\title{
Una experiencia de 'aprendizaje-servicio": fomentando el desarrollo de valores en estudiantes de odontología chilenos
}

\author{
Leonor Palomer, Paulina Humeres, Alejandra Sánchez, Soledad González, Antonieta Contreras
}

Introducción. Las universidades tienen el desafío trascendente de fomentar el desarrollo de valores en sus estudiantes. Existen metodologías educativas como 'aprendizaje-servicio' ( $A+S)$ que propician, con un objetivo docente, el contacto entre los alumnos y la sociedad.

Objetivo. Presentar la experiencia de incorporar A+S en un curso de primer año de la carrera de odontología en la Pontificia Universidad Católica de Chile y describir cómo este hecho influyó en la percepción de los estudiantes en relación con el desarrollo de determinados valores.

Sujetos y métodos. Se aplicó una encuesta anónima de siete preguntas abiertas a 60 alumnos de odontología de primer año. Los estudiantes autoevaluaron la experiencia vivida, las actividades, las metodologías de enseñanza-aprendizaje y lo que esto significó para su aprendizaje, especialmente respecto al fortalecimiento de actitudes y valores. Los comentarios cualitativos, recogidos por el cuestionario de autoevaluación, se integraron y sistematizaron a partir de categorías emergentes.

Resultados. Los estudiantes percibieron e identificaron los valores intencionados, las situaciones en las que se fomentaron, y también apreciaron el hecho de que durante su formación académica se enfrentaran a situaciones reales y concretas en las que pudieran desarrollar valores y actitudes.

Conclusiones. Incorporando la metodología A+S y propiciando espacios de reflexión se puede favorecer la formación de valores en los estudiantes de la carrera de odontología, aspecto que ellos consideran importante para su futuro desempeño como odontólogos.

Palabras clave. Aprendizaje-servicio. Odontología. Valores sociales.

Facultad de Medicina (L. Palomer, P. Humeres); Centro de Desarrollo Docente (A. Sánchez, S. González, A. Contreras); Pontificia Universidad Católica de Chile. Santiago de Chile, Chile.

Correspondencia:

Leonor Palomer. Facultad de Medicina. Pontificia Universidad Católica de Chile. Vicuña Mackenna, 4860. Campus San Joaquín Macul. Santiago de Chile, Chile.

E-mail:

Ipalomer@uc.cl

Conflicto de intereses: No declarado.

Competing interests: None declared.

(c) 2013 FEM

\section{A 'service-learning' experience: promoting the development of values in Chilean dental students}

Introduction. Universities face an important challenge in their aim to develop values in their students. On the other side, there are methodologies that promote, with an educational purpose, the interaction between students and different social actors. One of them is 'service-learning' $(\mathrm{S}+\mathrm{L})$.

Aim. To describe the experience of incorporating the $S+L$ methodology in a first-year course of the career of Dentistry at the Pontificia Universidad Católica de Chile and illustrate how this decision influenced the perception of students regarding the development of certain values.

Subjects and methods. Sixty students from this course answered an anonymous seven-question survey where they evaluated the whole experience and the methodology, as well as the activities performed and how this course had affected their perception of specific attitudes and values. The qualitative comments collected by this questionnaire were also analyzed and systematized.

Results. Students perceived and identified the mentioned values, as well as situations in which they were encouraged and appreciated the fact that during their education they were faced with real and concrete situations in which they could develop values and attitudes.

Conclusion. Incorporating the $\mathrm{S}+\mathrm{L}$ methodology and promoting opportunities for reflection can encourage the development of values in students of Dentistry, something they consider important for their future roles as dentists.

Key words. Dentistry. Service-learning. Social values. 


\section{Introducción}

Uno de los grandes desafíos en educación superior es lograr una formación holística en los estudiantes, donde el sello de calidad no esté dado sólo por sus capacidades en el área de los conocimientos y habilidades, sino también por una rigurosa formación que desarrolle valores y actitudes, asegurando así que se licenciarán profesionales íntegros, comprometidos socialmente y preparados para afrontar las necesidades de la población donde ejercerán su labor.

Las universidades tienen un papel importante en la transmisión de los principios éticos de las profesiones y deben ser un lugar en el que se aprendan valores y contravalores. Además de preocuparse por la calidad de la enseñanza, les corresponde conseguir una mayor inclusión social y formar titulados que actúen desde perspectivas orientadas al logro del bien común y de una sociedad más justa y democrática [1].

En esta línea, Navarro declara que 'para adquirir competencias que van a facilitar el ejercicio de la responsabilidad social en el ámbito profesional, se requiere que, durante la formación, los estudiantes adquieran valores y actitudes y que también tengan la oportunidad de practicar conductas socialmente responsables' [2].

Por su parte, los estudiantes también demandan de la universidad que esta ejerza su papel respecto al compromiso social. En el estudio 'Responsabilidad social y sistema universitario chileno: visión y expectativas de los jóvenes estudiantes' [3], los participantes señalaron, en su gran mayoría, que la universidad debía motivar e incentivar la participación de los estudiantes, así como propiciar un mayor contacto con la realidad a través de prácticas y salidas a terreno, con el fin de lograr una mayor conexión entre la teoría y la realidad. Los estudiantes universitarios esperan que la universidad se acerque al entorno en que convive, que genere profesionales que respondan a las necesidades de la sociedad actual, que ayude a enfrentarse a los problemas sociales, que incentive la participación de los alumnos y genere una cultura crítica en su interior.

Para cumplir con estos objetivos, los currículos deben contemplar una especial preocupación por los niveles de desarrollo con que cada curso contribuye a esta formación de valores y por el diseño de instrumentos que aseguren un proceso de evaluación de calidad, donde se recojan las consideraciones del docente y del estudiante. Además, deben cerciorarse de que las metodologías de enseñanza-aprendizaje favorezcan que el joven afronte situaciones relacionadas con el ámbito de las actitudes [4].
Dentro de las metodologías que favorecen el desarrollo de actitudes y valores, las prácticas docentes y el contacto con la comunidad, se destaca la estrategia denominada 'aprendizaje-servicio' $(A+S)$, que el Bennion Center ha definido como 'una metodología pedagógica basada en la experiencia, en la que estudiantes, profesores y una comunidad trabajan juntos para integrar y aplicar el conocimiento académico en escenarios reales y así atender las necesidades de la comunidad, logrando los objetivos de instrucción mediante la acción, la reflexión crítica y la investigación, preparando a los estudiantes para convertirse en miembros que contribuyan a una sociedad justa y democrática' [5].

La ejecución de la metodología $A+S$ no debe confundirse con la prestación de servicios voluntarios. Las actividades programadas deben tener relevancia y reconocimiento en términos académicos, suponer un aprendizaje en los estudiantes y contribuir a la mejora de la calidad de vida y del nivel de inclusión social en la población [1]. Una condición fundamental es que la realización del servicio compatibilice los objetivos académicos del curso con las necesidades sentidas de las comunidades.

Resultados de trabajos que evalúan la experiencia de incorporar la metodología de $\mathrm{A}+\mathrm{S}$ en el currículo de carreras de la salud como enfermería [6] muestran que, desde la perspectiva de los estudiantes, se comprueba un compromiso solidario con las personas más necesitadas, una actitud positiva hacia la apreciación de la diversidad y valores fundamentales como el respeto por la vida, veracidad y honestidad, entre otros. Los estudiantes destacan que la experiencia les permitió lograr autoeficacia, optimizar su identidad, incrementar habilidades en el trabajo en equipo, mejorar la comunicación y liderazgo, y una clara manifestación de desarrollo y maduración personales.

En el área odontológica, cuando se han desarrollado actividades educativas en contacto con una comunidad, se ha descrito que dichas prácticas mejoran las experiencias de aprendizaje de los participantes y presentan un enorme potencial para influir en los valores de equidad, de servicio público y colaboración por parte de los estudiantes [7].

Tomando en consideración lo señalado y como una forma de incentivar la formación de valores y actitudes en la carrera de odontología de la Pontificia Universidad Católica de Chile (PUC), se implementó la modalidad de $\mathrm{A}+\mathrm{S}$ en el curso denominado 'Fundamentos Odontológicos Integrados II'. Este curso se dictó durante el segundo semestre del año 2010, duró un semestre y estuvo dirigido a estudiantes de primer año de odontología. Para llevar a cabo 
el proyecto se contó con el apoyo del Centro de Desarrollo Docente de la misma universidad, que cuenta con profesionales preparados en la metodología que acompañaron y controlaron el proceso.

El objetivo principal del curso donde se aplicó la metodología es introducir al alumno en el conocimiento de la realidad de la salud bucal de la población chilena más vulnerable, considerando indicadores sociales, económicos y epidemiológicos, que le permitan diseñar y ejecutar una intervención educativa. Concomitantemente se pretende estimular en el estudiante el desarrollo de una conciencia de su papel social y el trabajo en equipo con liderazgo positivo.

Con la implementación de la metodología $A+S$ se buscó potenciar este objetivo, pues apunta a alcanzar tres objetivos fundamentales: un aprendizaje eficaz de los contenidos del curso, la entrega de un servicio de calidad y el desarrollo de valores. El curso propició el contacto precoz con una comunidad, donde los estudiantes desarrollaron labores de educación, pudieron comprender los determinantes sociales que afectan la salud oral y aplicaron sobre el terreno medidas y estrategias que abarcaban contenidos recibidos previamente de forma teórica.

En el programa del curso se explicitó que se pretendía desarrollar tres actitudes, que concuerdan además con el perfil declarado como ideal para un licenciado en la PUC: la capacidad de trabajo en equipo, la solidaridad y el respeto.

El curso, de 60 alumnos ( 45 mujeres y 15 varones), se dividió en grupos al azar formados por nueve estudiantes a cargo de un docente, los cuales se hicieron responsables de una comunidad específica (socio comunitario). Algunos trabajaron con niños preescolares, otros con padres, otros con adultos mayores y otros con las personas a cargo del cuidado de estos ancianos. Las personas participantes pertenecían a las comunas de La Pintana y Puente Alto, sectores ubicados en la periferia sur del Gran Santiago, donde la población es en su mayoría de nivel socioeconómico bajo y medio. Primero se realizó una visita de diagnóstico para recabar información y determinar qué aspectos referidos a salud oral eran significativos para estos socios comunitarios y, posteriormente, cada grupo diseñó una actividad educativa para disminuir los factores de riesgo que los afectaban.

Junto con la incorporación de $\mathrm{A}+\mathrm{S}$, se establecieron instancias de reflexión en las distintas etapas del curso y de evaluación temprana del proceso. La evaluación final fue realizada tanto por los estudiantes, con una autoevalución y coevaluación, como por los docentes y por las comunidades que reci-
Tabla I. Respuestas a la pregunta 1: 'en relación a tu experiencia, ¿qué valores o actitudes fueron promovidos en el curso?'.

\begin{tabular}{|c|c|c|}
\hline & Menciones & Ejemplos de comentarios \\
\hline Trabajo en equipo & $38 \%$ & $\begin{array}{l}\text { 'Lo más importante fue aprender a confiar en el trabajo } \\
\text { de otros'. 'Darse cuenta que uno necesita del otro' }\end{array}$ \\
\hline $\begin{array}{l}\text { Responsabilidad } \\
\text { social }\end{array}$ & $27 \%$ & $\begin{array}{l}\text { 'La importancia del servicio a otros en la formación } \\
\text { de una carrera de salud' }\end{array}$ \\
\hline Respeto & $18 \%$ & 'Respeto por la opinión de los demás, escuchar al otro' \\
\hline Comunicación & $8 \%$ & 'Aprender a compartir ideas, expresarnos y participar' \\
\hline $\begin{array}{l}\text { Actitudes hacia } \\
\text { el aprendizaje }\end{array}$ & $8 \%$ & $\begin{array}{l}\text { 'Constancia'. 'Esfuerzo'. 'Tolerancia'. 'Creatividad'. 'Autocrítica' } \\
\text { 'Valoración de las capacidades que tiene cada uno' }\end{array}$ \\
\hline
\end{tabular}

Tabla II. Respuestas a la pregunta 2: '¿en qué lo notaste? (situaciones de aprendizajes, ejemplos concretos, retroalimentaciones)'.

$$
\text { Menciones Ejemplos de comentarios }
$$

$\begin{array}{lll}\begin{array}{l}\text { Diseño y } \\ \text { metodología }\end{array} & 32 \% & \begin{array}{l}\text { 'El momento más claro fue al tener que realizar nuestra } \\ \text { presentación final, donde cada uno de nosotros tuvo que cumplir } \\ \text { con lo que le fue asignado para poder hacer un buen trabajo' }\end{array}\end{array}$

\begin{tabular}{lll}
\hline Trabajo con el & 'Estando en la intervención con el socio comunitario, con su \\
socio comunitario & $25 \%$ & $\begin{array}{l}\text { respuesta pude notar que con mínimas cosas y conocimientos } \\
\text { se les puede cambiar un hábito o actitud para mejorar su } \\
\text { calidad de vida' }\end{array}$
\end{tabular}

\begin{tabular}{|c|c|c|}
\hline Trabajo en equipo & $19 \%$ & $\begin{array}{l}\text { 'En las distintas actividades que tuvimos que realizar, se requería } \\
\text { de la participación y aporte de ideas de todos los integrantes del } \\
\text { grupo para hacer un trabajo constructivo' }\end{array}$ \\
\hline $\begin{array}{l}\text { Evaluación y } \\
\text { retroalimentación }\end{array}$ & $18 \%$ & $\begin{array}{l}\text { 'En la intervención misma y en las evaluaciones } \\
\text { entre compañeros y autoevaluación' }\end{array}$ \\
\hline $\begin{array}{l}\text { Trabajo con } \\
\text { los docentes }\end{array}$ & $5 \%$ & $\begin{array}{l}\text { 'La puntualidad de los docentes nos obligó a ser puntuales } \\
\text { a nosotros' }\end{array}$ \\
\hline
\end{tabular}

bieron el servicio. En relación a la experiencia de los estudiantes se enfatizó la medición de la percepción de los cambios respecto a actitudes como la solidaridad -también denominada 'responsabilidad social'-, el respeto y el trabajo en equipo. Para ello se diseñaron, de forma colaborativa entre los docentes y miembros del Centro de Desarrollo Docente, instrumentos de autoevaluación y se establecieron actividades que incentivaron la reflexión, como la lectura de artículos con desarrollo de ensayos, cuestionarios con preguntas abiertas sobre la experiencia y espacios de conversación y retroalimentación con los docentes. 
Tabla III. Respuestas a la pregunta 3: '¿qué tan de acuerdo estás con la siguiente afirmación?: la retroalimentación y comentarios recibidos por el profesor(es), en actividades realizadas y los trabajos que entregué, me permitieron identificar mis fortalezas y áreas a mejorar en relación a mis actitudes y valores'.

\begin{tabular}{lcl}
\hline Menciones & Ejemplos de comentarios \\
\hline $\begin{array}{l}\text { Totalmente } \\
\text { de acuerdo }\end{array}$ & $62 \%$ & $\begin{array}{l}\text { 'Me permitió darme cuenta de qué fortalezas y debilidades } \\
\text { tengo, para poder mejorarlas'. 'Fue muy importante la opinión y } \\
\text { retroalimentación de nuestro tutor para mejorar nuestros errores, } \\
\text { mediante una comunicación muy abierta y sincera' }\end{array}$ \\
\hline En acuerdo & $37 \% \quad \begin{array}{l}\text { 'La retroalimentación fue más dirigida hacia el grupo y no hacia } \\
\text { cada alumno individualmente'. 'En cierto modo sí, pero al momento } \\
\text { de la retroalimentación fue algo general y no personal' }\end{array}$ \\
\hline
\end{tabular}

$\begin{array}{lll}\text { En desacuerdo } & \begin{array}{l}\text { 'Creo que está bien que no entendamos lo que ustedes quisieron } \\ \text { decir, pero ustedes tampoco aceptan que no saben expresar lo } \\ \text { que querían decir' }\end{array}\end{array}$

Totalmente

en desacuerdo

$0 \%$

Tabla IV. Respuestas a la pregunta 4: 'a partir de la experiencia de servicio menciona los tres aprendizajes más importantes para ti'.

\begin{tabular}{lcl}
\hline & Menciones & $\begin{array}{l}\text { Ejemplos de comentarios } \\
\text { Trabajo en equipo }\end{array}$ \\
\hline $\begin{array}{l}\text { Responsabilidad social } \\
\text { 'Tolerancia a las diferentes ideas'. 'Saber escuchar atentamente } \\
\text { al resto' }\end{array}$ & $27 \%$ & $\begin{array}{l}\text { 'Darme cuenta de las diferentes realidades que existen y que } \\
\text { nuestro rol como futuros odontólogos es muy importante } \\
\text { y que debemos ser promotores de salud' }\end{array}$ \\
\hline $\begin{array}{l}\text { Planificación y aplicación } \\
\text { de una intervención }\end{array}$ & $17 \%$ & $\begin{array}{l}\text { 'La importancia de centrarse en un determinado objetivo, } \\
\text { delimitando bien las actividades para dicho objetivo' }\end{array}$ \\
\hline $\begin{array}{l}\text { Habilidades de } \\
\text { aprendizaje personal }\end{array}$ & $14 \%$ & $\begin{array}{l}\text { 'Aplicación de conceptos teóricos'. 'Disposición, entusiasmo } \\
\text { y organización' }\end{array}$ \\
\hline $\begin{array}{l}\text { Respeto } \\
\text { 'Respeto por la condición del otro, el contexto y la situación } \\
\text { en que vive' }\end{array}$ \\
\hline
\end{tabular}

Este estudio describe cómo la incorporación de $\mathrm{A}+\mathrm{S}$ en el primer año de la carrera de odontología en la PUC y la aplicación de instrumentos de autoevaluación y reflexión permitieron medir la experiencia que tuvo el curso para los estudiantes en cuanto a la percepción del desarrollo de valores.

\section{Sujetos y métodos}

Al término del curso, en el marco de la evaluación de la experiencia por parte de los estudiantes, se aplicó un instrumento de autoevaluación a los 60 alumnos que participaron en el programa 'Fundamentos Odontológicos Integrados II'. Hubo cuatro estudiantes que no terminaron el curso y abandonaron la carrera durante ese semestre.

Se usó una encuesta anónima de siete preguntas abiertas, que se entregó a cada uno de los integrantes de los grupos de trabajo de seminario. El profesor a cargo dio las instrucciones y respondió las preguntas de sus estudiantes. Al final de la aplicación del instrumento no se comentaron los resultados.

Las preguntas indagaban sobre la experiencia vivida, las actividades donde se promovían valores, las metodologías de enseñanza-aprendizaje incorporadas al curso y lo que esto significó para su aprendizaje, especialmente en relación al fortalecimiento de actitudes y valores. Los estudiantes podían mencionar libremente los aspectos que consideraban importantes para su formación y que eran autoevaluados.

Los resultados de la autoevaluación fueron analizados por el Centro de Desarrollo Docente, se integraron y sistematizaron los comentarios cualitativos a partir de categorías emergentes, recogidos por el cuestionario de autoevaluación, y se cuantificaron las menciones que se hacía en cada uno de los aspectos solicitados.

\section{Resultados}

La primera pregunta fue: 'en relación a tu experiencia, ¿qué valores o actitudes fueron promovidos en el curso?'. Los estudiantes realizaron libremente 120 menciones. Los valores mayoritariamente mencionados fueron: trabajo en equipo, responsabilidad social, respeto, comunicación y actitudes hacia el aprendizaje (Tabla I).

La segunda pregunta consultaba acerca de: ‘en qué lo notaste? (situaciones de aprendizaje, ejemplos concretos, retroalimentaciones)'. Los estudiantes mencionaron 93 situaciones. Las mayores referencias aludieron a: diseño y metodología del curso, trabajo con el socio comunitario, trabajo en equipo, instancias de evaluación y retroalimentación, y trabajo con los docentes (Tabla II).

La tercera pregunta planteaba: ‘qué tan de acuerdo estás con la siguiente afirmación?: la retroalimentación y comentarios recibidos por el profesor(es), en actividades realizadas y los trabajos que entregué, me permitieron identificar mis fortalezas y áreas a mejorar en relación a mis actitudes y valores'. Mayoritariamente, los estudiantes estuvieron totalmente de acuerdo (Tabla III). 
La consulta número cuatro pedía: 'a partir de la experiencia de servicio menciona los tres aprendizajes más importantes para ti'. Se obtuvieron 143 menciones por parte de los 60 estudiantes. Los aprendizajes más nombrados fueron: trabajo en equipo, responsabilidad social, planificación y aplicación de una intervención, habilidades de aprendizaje personal y respeto (Tabla IV).

La pregunta número cinco planteaba: 'en relación a tu aprendizaje de actitudes y valores desarrollado durante el curso, ¿qué harías diferente en tu proceso personal?', La mayoría respondió que haría diferencias en su trabajo personal, después se mencionó el servicio y el resto no cambiaría nada (Tabla V).

La sexta pregunta planteaba: 'el curso me aportó elementos nuevos sobre mi aprendizaje en actitudes y valores, ¿cuáles?'. Ante esto, los estudiantes mencionaron 75 nuevos elementos. Lo más referido fue la capacidad de trabajar en equipo, la responsabilidad social, el respeto, el autoconocimiento y refuerzo de habilidades personales, el conocimiento de un modelo de planificación y la comunicación efectiva (Tabla VI).

Finalmente, la pregunta siete decía: ‘qué nivel de importancia le asigna a la formación en actitudes y valores en la carrera?.' La gran mayoría le asignó una alta importancia (Tabla VII).

\section{Discusión}

De acuerdo con los resultados obtenidos, la experiencia de incorporar una actividad de servicio en una comunidad utilizando la metodología de $\mathrm{A}+\mathrm{S}$ y permitiendo los espacios de reflexión, favorecería la percepción del desarrollo de valores en los estudiantes. Complementariamente, la modalidad de trabajo en pequeños grupos ayudaría a promover actitudes positivas que son reconocidas por los participantes.

Los valores que se fomentaron en el curso (respeto, solidaridad o responsabilidad social y trabajo en equipo) son visualizados por los estudiantes e incluso reconocen la promoción de otros valores y actitudes más allá de los propuestos originalmente en el programa de curso, como la comunicación y actitudes hacia el aprendizaje. Por tanto, al diseñar situaciones de aprendizaje se puede promover el desarrollo de valores, hecho que los estudiantes aprecian $\mathrm{y}$ al cual asignan un alto valor en su formación.

Estos resultados concuerdan con lo indicado en publicaciones sobre la implementación de $A+S$, que destacan la obtención de aprendizajes que fomentan y contribuyen al desarrollo de aspectos no cognitivos en los estudiantes, particularmente en áreas
Tabla V. Respuestas a la pregunta 5: 'en relación a tu aprendizaje de actitudes y valores desarrollado durante el curso, ¿qué harías diferente en tu proceso personal?'.

\begin{tabular}{lcl}
\hline Menciones & Ejemplos de comentarios \\
\hline Trabajo personal & $78 \%$ & $\begin{array}{l}\text { 'Mejoraría la capacidad de compromiso y organización personal'. } \\
\text { 'Ver más mis errores, aceptarlos y estar más abierto a opiniones'. }\end{array}$ \\
\hline $\begin{array}{l}\text { El trabajo en } \\
\text { relación al servicio }\end{array}$ & $16 \%$ & $\begin{array}{l}\text { 'Reconocer y disminuir los prejuicios, por ejemplo, habría } \\
\text { Ilegado con una mentalidad más abierta'. 'Me involucraría } \\
\text { más en el trabajo grupal' }\end{array}$ \\
\hline Nada & $5 \%$ & \\
\hline
\end{tabular}

Tabla VI. Respuestas a la pregunta 6: 'el curso me aportó elementos nuevos sobre mi aprendizaje en actitudes y valores, ¿cuáles?'.

\begin{tabular}{|c|c|c|}
\hline & Menciones & Ejemplos de comentarios \\
\hline $\begin{array}{l}\text { Capacidad de } \\
\text { trabajar en equipo }\end{array}$ & $25 \%$ & $\begin{array}{l}\text { 'Aprendí que aprendo mejor en grupo y que debo tener más } \\
\text { tolerancia para relacionarme con otros en un trabajo grupal' }\end{array}$ \\
\hline $\begin{array}{l}\text { Responsabilidad } \\
\text { social }\end{array}$ & $25 \%$ & $\begin{array}{l}\text { 'Responsabilidad social, nunca habría realizado ningún tipo } \\
\text { de actividad en beneficio de la sociedad, es muy gratificante } \\
\text { poder ayudar realmente a otra persona' }\end{array}$ \\
\hline Respeto & $23 \%$ & 'Noción de la importancia del respeto en las relaciones humanas' \\
\hline $\begin{array}{l}\text { Autoconocimiento } \\
\text { y refuerzo de } \\
\text { habilidades personales }\end{array}$ & $12 \%$ & $\begin{array}{l}\text { 'Descubrí que puedo llegar a ser muy responsable, } \\
\text { que tengo capacidad de liderazgo y de empatía' }\end{array}$ \\
\hline $\begin{array}{l}\text { Conocimiento de un } \\
\text { modelo de planificación }\end{array}$ & $8 \%$ & $\begin{array}{l}\text { 'Aplicar un modelo lógico y sistemático que atiende a las } \\
\text { personas en su contexto y a partir de las capacidades propias' }\end{array}$ \\
\hline $\begin{array}{l}\text { Comunicación } \\
\text { efectiva }\end{array}$ & $7 \%$ & $\begin{array}{l}\text { 'Me enseñó a expresarme, a dar mis ideas, a dar mis opiniones, } \\
\text { a organizarnos y expresar mi creatividad, sobre todo en la } \\
\text { intervención' }\end{array}$ \\
\hline
\end{tabular}

relacionadas con la responsabilidad cívica, como son la conciencia de las necesidades de una comunidad, la apreciación de las diferencias entre las personas, la empatía por los demás y la capacidad para relacionarse con otros. Tales estudios concluyen que esta metodología puede hacer una importante contribución a la evaluación de los resultados de aprendizaje de los alumnos en relación con la adquisición de actitudes y creencias que son antecedentes evidentes de responsabilidad cívica, aspecto que reafirma este trabajo [8].

Por otra parte, la elevada importancia que los estudiantes otorgan a la formación en actitudes y valores en la carrera y las respuestas obtenidas en cuanto al desarrollo de una conciencia social permiten 
Tabla VII. Respuestas a la pregunta 7: '¿qué nivel de importancia le asigna a la formación en actitudes y valores en la carrera?'.

\section{Porcentaje Ejemplos de comentarios}

'En un profesional de la salud, ser íntegro y desarrollar todo tipo de valores y
actos, ya que se trabaja con personas, no sólo con enfermedades, siendo esto lo
más enriquecedor de la carrera'. 'Permitió desarrollar en nosotros actitudes
que no habíamos desarrollado antes'. 'Trabajos como éste no sólo te forman
como profesional, sino que lo hacen como persona'. 'Creo muy valorable el
hecho de que existan trabajos con compromiso social'. 'Enseña sobre la realidad
Alto nuestro país y nuestra responsabilidad y compromiso con la sociedad'. 'No
sólo seremos doctores, debemos ser personas integrales en todos los aspectos
de nuestra vida'. 'En el colegio no estaba acostumbrado a trabajar en equipo,
generalmente hacía la mayor parte del trabajo; aquí pudimos dividir los
diferentes aspectos de los trabajos y funcionamos como grupo'

\begin{tabular}{lll}
\hline Regular & $7 \%$ & $\begin{array}{l}\text { 'Se trata de una buena manera que el paciente es un todo y no sólo una } \\
\text { boca'. 'Creo que es un modelo excelente de trabajo; sin embargo, deberían } \\
\text { haber más intervenciones' }\end{array}$ \\
\hline Bajo & $0 \%$ & \\
\hline Nulo & $0 \%$ & \\
\hline
\end{tabular}

aventurar que algunos de los involucrados en esta experiencia continuarán participando en actividades similares. Según trabajos como el de Gray et al [9], los estudiantes que han tenido la oportunidad de realizar una experiencia de $\mathrm{A}+\mathrm{S}$ continuarán participando posteriormente en trabajos voluntarios y tendrán un papel más activo en ayudar a las comunidades frente a los problemas sociales. En este estudio, los estudiantes reconocen que haber participado en un curso de $\mathrm{A}+\mathrm{S}$ les otorgó habilidades para la vida, incluyendo las aptitudes interpersonales y el conocimiento de personas con una realidad diferente a la suya.
En síntesis, y tomando en cuenta el importante papel que desempeña la educación superior en la formación integral de personas, los resultados muestran que resulta de gran trascendencia enfrentar al estudiante con situaciones de aprendizaje que le permitan desarrollar valores, actitudes y estilos de comportamiento. En este sentido, la incorporación de metodologías de enseñanza-aprendizaje, como $\mathrm{A}+\mathrm{S}$, favorece plenamente dicho objetivo y la evaluación del proceso permite recibir retroalimentación por parte de los involucrados, dimensionando el logro de la tarea.

\section{Bibliografía}

1. Martínez M. Aprendizaje-servicio y responsabilidad social de las universidades. Barcelona: Octaedro y Fundació Jaume Bofill; 2008.

2. Navarro G. Comportamiento socialmente responsable. 2005. URL: http://lahoradelaetica.files.wordpress.com/2008/02/ comportamiento-socialmente-responsable.pdf.

3. Giacomán C, Opazo M. Responsabilidad social y sistema universitario chileno: visión y expectativas de los jóvenes estudiantes. Santiago de Chile: Pontificia Universidad Católica de Chile, Instituto de Sociología y Fundación Participa; 2002.

4. Oliver R, Kersten H, Vinkka-Puhakka H, Alpasan G, Bearn D, Cema I, et al. Curriculum structure: principles and strategy. Eur J Dent Educ 2008; 12: 74-84.

5. Grauerholz L. Teaching holistically to achieve deep learning. College Teaching 2001; 49: 44-50.

6. Arratia-Figueroa A. Ética, solidaridad y 'aprendizaje-servicio' en la educación superior. Acta Bioeth 2008; 14: 61-7.

7. Straus RP, Stein MB, Edwards J, Nies KC. The impact of community-based dental education on students. J Dent Educ 2010; 74: S42.

8. Weglarz S, Seybert J. Participant perceptions of a community college service-learning program. Community College Journal of Research \& Practice 2004; 28: 123-32.

9. Gray MJ, Ondaatje EH, Zakaras L. Combining service and learning in higher education: summary report. Santa Monica, CA: RAND Education; 1999. URL: http://www.dtic.mil/ cgi-bin/GetTRDoc?AD=ADA380605\&Location $=\mathrm{U} 2 \&$ doc $=$ GetTRDoc.pdf. 